\title{
THE HYBRID METHOD OF FINDING THE NATURAL FREQUENCY SPECTRUM FOR QUASI-FRACTAL PLATE BANDS
}

\author{
Artur Wirowski \\ Department of Structural Mechanics, Lodz University of Technology \\ Łódź, Poland \\ artur.wirowski@p.lodz.pl
}

\begin{abstract}
The work deals with the issues of modelling of multiscale composites. A group of composite structures with a quasi-fractal geometry has been defined. In order to model their dynamic properties, a simple engineering method has been proposed. It takes into account not only the parameters and proportions of the components of the composite, but also their mutual arrangement. The proposed method is demonstrated on several examples and its accuracy has been compared with the finite element method and the homogenization method.
\end{abstract}

Keywords: composite plate, quasi-fractal plates, tolerance averaging technique, hybrid methods, vibrations

\section{Introduction}

\subsection{Discussion of the problem}

The problem of searching the entire spectrum of natural frequencies for composites is a difficult issue both numerically and analytically. The internal microstructure of the composite has an increasing influence on the sequential natural frequencies. The sequential forms of natural vibrations are usually periodic or quasi-periodic. The length of their periodicity is of the order of the size of the characteristic microstructure of the examined structure.

The majority of the approximate methods requires the size of the concerned body to be much larger than the size of its microstructure [1] or the estimated displacement field to be described by a slowly varying function in an area of the microstructure [2]. These conditions preclude the direct application of approximate methods to the considered issue.

Composite materials are increasingly used in the modern construction industry. Very often their structure is inspired by nature [3] or complicated mathematical solutions [4]. In this work, the band plate of the quasi-fractal structure will be considered. It can be used for example as roof panels which combine functionality 
and aesthetics. In such applications, different materials are used, most often light transmission glass or a steel or an aluminum matrix to provide durability and strength.

\subsection{Definition of quasi-fractal materials}

Let $\Omega \in R^{3}$ be a body occupying a limited area. In this body we determine a subdivision $\Lambda \subset \Omega$, which is characteristic for a given structure, and we call it a basic cell. Let $Z=\left(z_{1}, z_{2}, z_{3}\right) \in \Lambda$ and $Y=\left(y_{1}, y_{2}, y_{3}\right) \in \tilde{\Lambda}_{i}$ be points in the physical space $R^{3}$. Let $F(Z)$ be a function that defines all the local physical parameters of the body at the point $Z$. By $\tilde{\Lambda}_{i} \subset \Omega$ we denote the similar and scaled area in relation to the $\Lambda$ satisfying the following conditions:

$$
\begin{gathered}
\tilde{\Lambda}_{i} \cap \Lambda=0, \\
Y=\kappa_{i} Z \Leftrightarrow F(Y)=F(Z),
\end{gathered}
$$

where $\kappa_{i} \in R$ is a number representing the similarity scale between cells $\Lambda$ and $\tilde{\Lambda}_{i}$.

The body will be called quasi-fractal if we can find such a basic cell $\Lambda$ and we choose such a series of similarity scales $\kappa_{i}$ and we define such a series of subareas $\tilde{\Lambda}_{i}$ for that the following conditions are satisfied

$$
\Lambda \bigcup \bigcup_{i} \tilde{\Lambda}_{i} \equiv \Omega
$$

This means that the whole composite can be divided into cells similar to each other with similarity scales $\kappa_{i}$. The examples of such structures are shown in Figure 1. In contrast to the standard fractal definition, such structures are always finite and in practice they can be used to create engineering constructions.
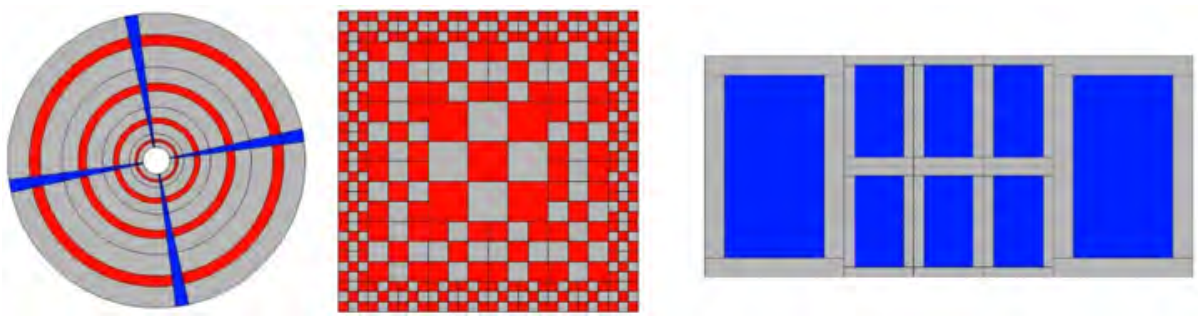

Fig. 1. Examples of quasi-fractal structures 
Let us note that, in accordance with the above definition, a quasi-fractal structure can also be built in a one-dimensional space, which is not possible with a classic fractal structure and that a periodic structure is a special case of a quasi-fractal structure if $\underset{i}{\forall} \kappa_{i} \equiv 1$.

\subsection{Subject, purpose and scope of the paper}

The subject of this paper is a quasi-fractal plate band. The aim of this work is to establish a simple and as precise as possible analytical method to find many natural frequencies of such a band. It is based on finding a replacement property of the plate band taking into account basic cell properties and the structure of the whole composite. As a result, every quasi-fractal structure can be reduced to the corresponding periodic structure by adopting $\kappa_{i} \equiv 1$. This method allows one to consider the cases in which the microstructure size $\lambda$ can be comparable with dimension which characterizes the natural vibration form of the structure. The proposed method, called a hybrid method later in the work, is illustrated with a few examples in Section 3. The obtained results will be compared with numerical solutions obtained by using the finite element method (FEM) in the Autodesk Simulation Multiphysics program of 2013. Its accuracy will be confronted with the classic methods of asymptotic homogenization.

\section{Derivation of replacement properties of the plate band taking into account the structure of the material}

\subsection{Assumptions}

We assume that the frequency of free vibrations of the plate band of the quasifractal construction $\Omega_{p}$ depends mainly on the structure and the material properties of its basic cell, and the macroscopic dimensions of the band and its support conditions, and to a lesser degree, on the distribution of cells and their mutual scale similarities. Therefore the following reasoning has been carried out. Let us take the band plate of the quasi-fractal construction and the corresponding band plate of the periodic construction $\Omega_{p}$ constructed in such a way that the basic cell of the band plate of the quasi-fractal construction is identical to the periodicity plate band, and a length $L$ of the periodic plate band could be freely adopted so as to satisfy the condition $L>>\lambda$, where $\lambda$ is the size of the periodicity cell. For the above plate band $\Omega_{p}$ we determine the averaged replacement material properties taking into account the influence of the microstructure size. We assume that the obtained averaged moduli describe the properties of the corresponding plate bands of the quasi-fractal construction $\Omega$ with good approximation. 


\subsection{Tolerance averaging technique}

The replacement plate band of the periodic structure $\Omega_{p}$ will be modeled using the tolerance averaging technique (TAT). It is a known method of modelling microstructured materials which was proposed by Woźniak and Wierzbicki in [6] and it has been used with success in many engineering applications for materials of periodic structure [7] and functionally variable structure $[8,9]$. It allows one to consider the effect of the material microstructure on the macroscopic properties of the composite [10].

We mention some basic concepts of this technique, e.g. averaging operator, a tolerance parameter, a tolerance periodic function, a slowly varying function, a highly oscillating function. These definitions will hold for the one-dimensional case. The important concept of the TAT is the averaging of an arbitrary integrable function $f(\cdot)$ over the cell $\Delta\left(x_{\alpha}\right)[2]$ :

$$
<f>(x)=\frac{1}{\lambda} \int_{x-\lambda / 2}^{x+\lambda / 2} f(x) d x .
$$

The fundamental concept of the tolerance averaging technique is that values of functions that belong to region $\Pi$ can be determined only within a certain accuracy $\delta$. Let $\delta$ stand for an arbitrary positive number and $X$ be a linear normed space. The tolerance relation $\approx$ for a certain $\delta$ is defined by

$$
\left(\forall\left(x_{1}, x_{2}\right) \in X^{2}\right)\left[x_{1} \approx x_{2} \Leftrightarrow\left\|x_{1}-x_{2}\right\|_{X} \leq \delta\right],
$$

where $\delta$ is said to be the tolerance parameter.

Let $\partial^{k} f$ be the $k$ gradient of function $f=f(\mathbf{x}), \mathbf{x} \in \Pi, k=0,1, \ldots, \alpha,(\alpha \geq 0)$, $\partial^{0} f \equiv f$. Function $f \in H^{\alpha}(\Pi)$ will be called the tolerance periodic function (with respect to cell $\Delta$ and tolerance parameter $\delta$ ), $f \in T P_{\delta}^{\alpha}(\Pi, \Delta)$, if for $k=0,1, \ldots, \alpha$, the following conditions hold $(\forall \mathbf{x} \in \Pi)\left(\exists \tilde{f}^{(k)}(\mathbf{x}, \cdot) \in H^{0}(\Delta)\right)$ $\left[\left\|\left.\partial^{k} f\right|_{\Pi_{\mathbf{x}}}(\cdot)-\tilde{f}^{(k)}(\mathbf{x}, \cdot)\right\|_{H^{0}\left(\Pi_{\mathbf{x}}\right)} \leq \delta\right]$,

$$
\int_{\Delta(\cdot)} \tilde{f}^{(k)}(\cdot, y) d y \in C^{0}(\bar{\Pi})
$$

Function $\tilde{f}^{(k)}(\mathbf{x}, \cdot)$ is referred to as the periodic approximation of $\partial^{k} f$ in $\Delta(\mathbf{x}), \mathbf{x} \in \Pi, k=0,1, \ldots, \alpha$. Function $F \in H^{\alpha}(\Pi)$ will be called the slowly varying function (with respect to the cell $\Delta$ and tolerance parameter $\delta$ ), $F \in S V_{\delta}^{\alpha}(\Pi, \Delta)$, if $F \in T P_{\delta}^{\alpha}(\Pi, \Delta)$,

$$
(\forall \mathbf{x} \in \Pi)\left[\left.\tilde{F}^{(k)}(\mathbf{x}, \cdot)\right|_{\Delta(\mathbf{x})}=\partial^{k} F(\mathbf{x}), \quad k=0, \ldots, \alpha\right] .
$$


It can be observed that the periodic approximation $\tilde{F}^{(k)}$ of $\partial^{k} F(\cdot)$ in $\Delta(\mathbf{x})$ is a constant function for every $\mathbf{x} \in \Pi$. If $F \in S V_{\delta}^{\alpha}(\Pi, \Delta)$ then

$$
p=0
$$

Function $\varphi \in H^{\alpha}(\Pi)$ will be called the highly oscillating function (with respect to the cell $\Delta$ and tolerance parameter $\delta), \varphi \in H O_{\delta}^{\alpha}(\Pi, \Delta)$, if $\varphi \in T P_{\delta}^{\alpha}(\Pi, \Delta)$,

$$
\forall F \in S V_{\delta}^{\alpha}(\Pi, \Delta)\left(f \equiv \varphi F \in T P_{\delta}^{\alpha}(\Pi, \Delta)\right),
$$

and for $k=1, \ldots, \alpha$ these functions satisfy the conditions

$$
\begin{gathered}
(\forall \mathbf{x} \in \Pi)\left[\left.\tilde{\varphi}^{(k)}(\mathbf{x}, \cdot)\right|_{\Delta(\mathbf{x})}=\partial^{k} \tilde{\varphi}(\mathbf{x})\right], \\
\left.\tilde{f}^{(k)}(\mathbf{x}, \cdot)\right|_{\Delta(\mathbf{x})}=\left.F(\mathbf{x}) \partial^{k} \tilde{\varphi}(\mathbf{x})\right|_{\Delta(\mathbf{x})} .
\end{gathered}
$$

If $\alpha=0$, then we denote $\tilde{f} \equiv \tilde{f}^{(0)}$.

We denote by $\phi(\cdot)$ a highly oscillating function, $\phi \in H O_{\delta}^{2}(\Pi, \Delta)$, defined on $\Pi$, continuous together with gradient $\partial^{1} \phi$. Let its gradient $\partial^{2} \phi$ be piecewise continuous and bounded. Function $\phi(\cdot)$ will be called the fluctuation shape function of the 2 nd kind, if it depends on $\lambda$ as a parameter and satisfies the conditions

$$
\partial^{k} \phi \in O\left(\lambda^{\alpha-k}\right) \text { for } k=1, \ldots, \alpha, \alpha=2
$$

$2^{\circ}$

$$
<\phi>(\mathbf{x}) \approx 0 \text { for every } \mathbf{x} \in \Pi_{\Delta} .
$$

The set of all fluctuation shape functions of the 2nd kind is denoted by $F S_{\delta}^{2}(\Pi, \Delta)$. Condition $\left(2^{\circ}\right)$ can be replaced by $\left\langle\mu \phi>(\mathbf{x}) \approx 0\right.$ for every $\mathbf{x} \in \Pi_{\Delta}$, where $\mu>0$ is a certain tolerance periodic function.

In order to derive averaged model equations, we apply the tolerance averaging approach. The first assumption in the tolerance modelling is the micro-macro decomposition of the displacement field

$$
w(x, t)=w^{0}(x, t)+h^{A}(x) V_{A}(x, t), A=1, \ldots, N
$$

The modelling assumption states that $w^{0}(x, t), V_{A}(x, t)$ are slowly varying functions. Functions $w^{0}(x, t) \in S V_{\delta}^{2}(\Omega, \Delta), V_{A}(x, t) \in S V_{\delta}^{2}(\Omega, \Delta)$ are the basic unknowns of the modelling problem. Functions $h^{A}(\cdot)$, so-called fluctuation shape functions, are known and dependent on the microstructure length parameter $\lambda$. 
Let $\tilde{h}^{A}(\cdot), \partial_{1} \tilde{h}^{A}(\cdot)$ stand for the periodic approximation of $h^{A}(\cdot), \partial_{1} h^{A}(\cdot)$ in $\Delta$, respectively. Due to the fact that $w(x, t)$ are tolerance periodic functions, it can be observed that the periodic approximation of $w_{h}(x, t)$ and $\partial_{\beta} w_{h}(x, t)$ in $\Delta\left(\xi^{1}\right)$, $\xi^{1} \in \Pi$ has the form

$$
\begin{aligned}
& w_{h}(y, t)=w^{0}(x, t)+h^{A}(y) V_{A}(x, t), \\
& \partial_{\beta} w_{h}(y, t)=\partial_{\beta} w^{0}(x, t)+\partial_{1} h^{A}(y) V_{A}(x, t)+h^{A}(y) \partial_{2} V_{A}(x, t), \\
& \dot{w}_{h}(y, t)=\dot{w}^{0}(x, t)+h^{A}(y) \dot{V}_{A}(x, t),
\end{aligned}
$$

for every $x \in \Pi$, almost every $y \in \Delta(x)$ and every $t \in\left(t_{0}, t_{1}\right)$.

TAT also defines many assertions. For the purpose of this paper we quote the most important:

$$
\begin{aligned}
& \langle\phi F\rangle(x) \cong\langle\phi\rangle(x) F(x), \\
& \langle\phi \nabla F\rangle(x) \cong-\langle F \nabla \phi\rangle(x), \\
& \langle\nabla(F \phi)\rangle(x) \cong\langle F \nabla \phi\rangle(x),
\end{aligned}
$$

where $\phi \in H O_{\delta}^{2}(\Pi, \Delta)$ and $F \in S V_{\delta}^{\alpha}(\Pi, \Delta)$.

\subsection{Derivation of the replacement properties of the composite taking into account its structure}

The starting point of the modelling procedure is the thin plate equation according to Kirchhoff's theory which for the plate band can be written as

$$
\left(D w_{, 11}\right)_{, 11}+\mu \ddot{w}=p
$$

where: $D=\frac{E h^{3}}{12\left(1-v^{2}\right)}-$ stiffness of the plate, $w$ - deflection of the plate, $\mu=\rho h$ plate mass per unit $\left[\mathrm{kg} / \mathrm{m}^{2}\right], p$ - external load.

We assume the decomposition of the displacement field in the form:

$$
w=w^{0}+V g .
$$

By substituting (18) into (17), and transforming it by using the previously mentioned theorems of TAT, the first equation of the model can be obtained

$$
\langle D\rangle w_{, 111}^{0}+\left\langle D g_{, 11}\right\rangle V_{, 11}+\langle\mu\rangle \ddot{w}^{0}=\langle p\rangle \text {. }
$$


The second equation has been formulated through the orthogonalization method by multiplying equation (17) by a test function $g$, and then by averaging and transforming it, we obtain the following form

$$
-\left\langle D g_{, 11}\right\rangle w_{, 11}^{0}-\left\langle D g_{, 11} g_{, 11}\right\rangle V+\langle\mu g g\rangle \ddot{V}=\langle p g\rangle \text {. }
$$

Equations (19) and (20) form the equation system of the tolerance averaging technique model. We assume that there is no external force and reduce the problem to the free vibration

$$
V(x, t)=e^{i \omega t} \tilde{V}(x), w^{0}(x, t)=e^{i \omega t} \tilde{w}^{0}(x), p=0
$$

Moreover, assuming that the periodic plate band $\Omega_{p}$ can be built freely to make its length sufficiently large in relation to the size characterizing the microstructure, we can conclude that the microstructure goes to zero $\lambda \rightarrow 0$. Therefore the model equations are simplified to the form:

$$
\begin{gathered}
\langle D\rangle \tilde{w}_{, 111}^{0}+\left\langle D g_{, 11}\right\rangle \tilde{V}_{, 11}-\omega^{2}\langle\mu\rangle \tilde{w}^{0}=0 \\
\left\langle D g_{, 11}\right\rangle \tilde{w}_{, 11}^{0}+\left\langle D g_{, 11} g_{, 11}\right\rangle \tilde{V}=0 .
\end{gathered}
$$

From the second equation we can determine the unknown $\tilde{V}$ and substitute it into the first equation to receive only one equation defining the vibration of such a structure:

$$
\left(\langle D\rangle-\frac{\left\langle D g_{, 11}\right\rangle^{2}}{\left\langle D g_{, 11} g_{, 11}\right\rangle}\right) \tilde{w}_{, 1111}^{0}-\omega^{2}\langle\mu\rangle \tilde{w}^{0}=0
$$

When comparing this equation with the classic equation that describes the vibrations of a homogeneous plate band, we can define the replacement moduli characterizing the analyzed structure:

$$
D_{e f f}=\langle D\rangle-\frac{\left\langle D g_{, 11}\right\rangle^{2}}{\left\langle D g_{, 11} g_{, 11}\right\rangle}, \quad \mu_{e f f}=\langle\mu\rangle
$$

where

$$
\langle D\rangle=\frac{1}{\lambda} \int_{\lambda} D d x,\left\langle D g_{, 11}\right\rangle=\frac{1}{\lambda} \int_{\lambda} D g_{, 11} d x,\langle D\rangle=21 \mathrm{MNm},\langle\mu\rangle=\frac{1}{\lambda} \int_{\lambda} \mu d x
$$

These moduli are used later to determine the substitute material properties of a quasi-fractal plate band taking into account its basic cell construction. 


\subsection{Replacement material properties in the asymptotic homogenization}

The obtained effective moduli will be compared with standard solutions derived from asymptotic homogenization. On the basis of this theory, the effective material properties of the medium can be defined either in advance or afterwards respectively as the arithmetic and harmonic average, accordingly, taking into account the amount of the individual components in the composite.

$$
\left(f_{1} K_{1}^{-1}+f_{2} K_{2}^{-1}\right)^{-1}=K^{h} \leq K_{i j}^{e f f} \leq f_{1} K_{1}+f_{2} K_{2}
$$

This dependency will be used to determine the accuracy of the numerical results obtained from the proposed model. Finally, the value of $K^{\text {eff }}$ will be calculated as the arithmetic average of $K^{h}$ and $K^{a}$.

\section{Numerical examples}

\subsection{Material and geometrical data}

In order to illustrate the proposed method, in Table 1, some numerical examples were shown. Physical properties of the materials used are shown in Table 1.

Physical properties of materials used in the examples

\begin{tabular}{|c|c|c|c|c|}
\hline & & $\mathrm{E}[\mathrm{GPa}]$ & $\rho\left[\mathrm{kg} / \mathrm{m}^{3}\right]$ & $v[-]$ \\
\hline Aluminium & & 370 & 3960 & 0.22 \\
\hline Steel & & 207 & 7855 & 0.3 \\
\hline Glass & & 138 & 8360 & 0.317 \\
\hline
\end{tabular}

The same calculation methods are assumed in all three examples. The finite element method implemented in the Autodesk Simulation Multiphysics Program of 2013 has been used as reference to the calculated frequency values. Next, a few selected vibration frequencies and the corresponding vibration forms obtained in this program are presented.

The following notation is introduced: $\omega_{\mathrm{FEM}}$ is the reference frequency calculated from FEM, the vibration $\omega_{\mathrm{h}}$ is frequency obtained from analytical formulas (27), (28) and (31), respectively. The dependence of the accuracy of the solution obtained from the proposed method (continuous line) and asymptotic homogenization methods (dashed line), depending on the number of the subsequent values of free vibrations will be shown in Figures 4, 7 and 9 respectively for every example. 


\subsection{Quasi-fractal symmetrical structure - Example 1}

The first example is a plate band fully fixed on both sides, made from glass and aluminum as shown in Figure 2. The overall length of the band is $L=10 \mathrm{~m}$, the thickness $h=0.1 \mathrm{~m}$.

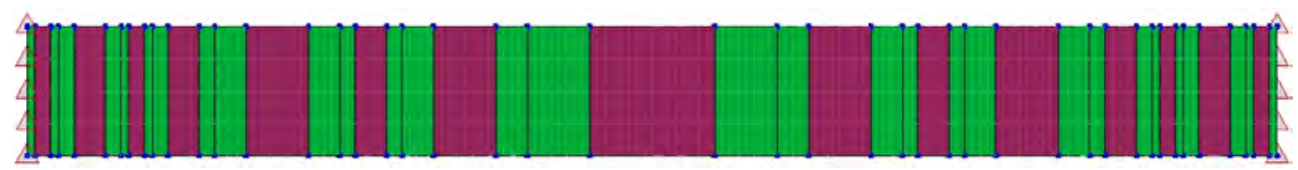

Fig. 2. Construction of the plate band from Example 1

For the above basic cell a symmetrical shape function has been taken. The effective properties of the plate band are defined by formulas (24) as:

$$
\begin{gathered}
g=\cos \left(\frac{2 \pi x}{\lambda}\right),\left\langle D g_{, 11}\right\rangle=-13 \frac{\mathrm{MN}}{\mathrm{m}},\left\langle D g_{, 11} g_{, 11}\right\rangle=64 \frac{\mathrm{MN}}{\mathrm{m}^{3}},\langle D\rangle=21 \mathrm{MNm}, \\
\langle\mu\rangle=616 \frac{\mathrm{kg}}{\mathrm{m}^{2}} .
\end{gathered}
$$

The plate band is fixed on both sides:

$$
\left.\tilde{w}^{0}\right|_{x=a}=0,\left.\frac{\partial \tilde{w}^{0}}{\partial x}\right|_{x=a}=0,\left.\tilde{V}\right|_{x=a}=0,\left.\frac{\partial \tilde{V}}{\partial x}\right|_{x=a}=0, a=\{0, L\}
$$

We use the classic solution derived for a homogeneous plate band:

$$
\omega=\frac{(2 k+1)^{2} \pi}{8 L^{2}} \sqrt{\frac{D_{e f f}}{\mu_{e f f}}}
$$

In Figure 3, we can see the best estimate of the value for the selected number of natural frequencies and their corresponding forms.

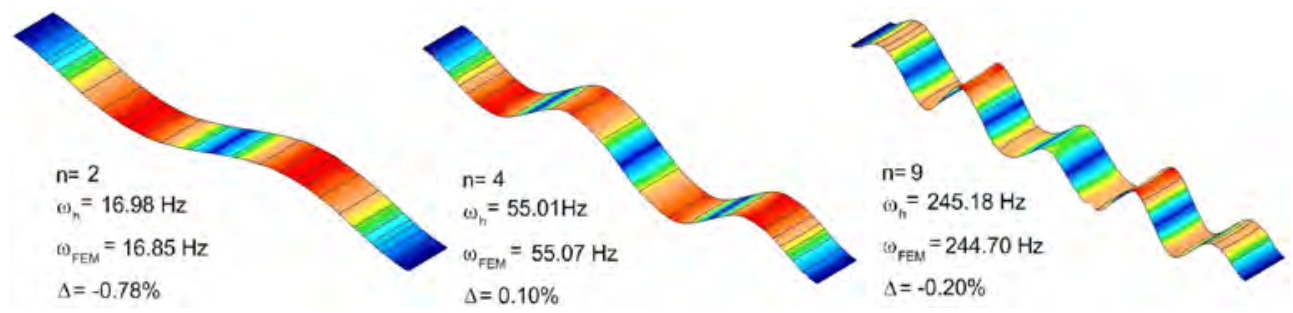

Fig. 3. Selected forms of free vibrations for Example 1 
In Figure 4 we show the accuracy of approximation for the first 17 natural frequencies. We can see that the classic asymptotic homogenization method underestimates the results, while the accuracy of solutions of the proposed hybrid method oscillates within the range of $+/-7 \%$.

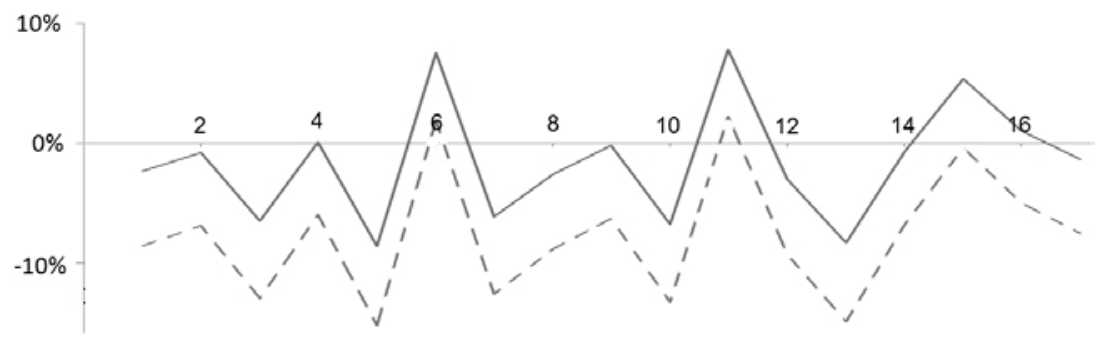

Fig. 4. Accuracy of solutions obtained from the proposed method (continuous line) and the asymptotic method (dashed line) in relation to the FEM

\subsection{Quasi-fractal antisymmetrical structure - Example 2}

The second example is a left-fixed bracket plate bands made of glass and aluminum as shown in Figure 5. The overall length of the band $L=20 \mathrm{~m}$, the thickness $h=0.1 \mathrm{~m}$.

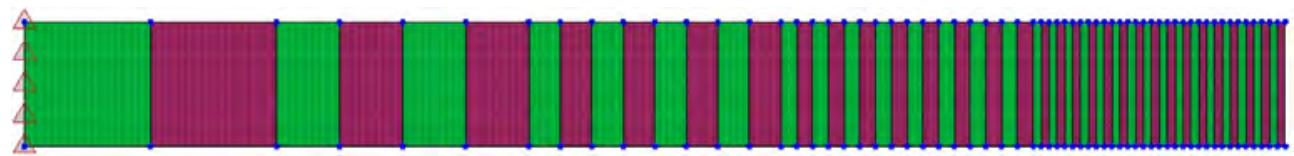

Fig. 5. Construction of the plate band from Example 2

For such a proposed basic cell geometry an antisymmetrical shape function has been taken in relation to the unit cell. The effective properties of the plate band are defined by formulas (24):

$$
\begin{gathered}
g=\sin \left(\frac{2 \pi x}{\lambda}\right),\left\langle D g_{, 11}\right\rangle=-52 \frac{\mathrm{MN}}{\mathrm{m}},\left\langle D g_{, 11} g_{, 11}\right\rangle=1025 \frac{\mathrm{MN}}{\mathrm{m}^{3}},\langle D\rangle=21 \mathrm{MNm}, \\
\langle\mu\rangle=616 \frac{\mathrm{kg}}{\mathrm{m}^{2}} .
\end{gathered}
$$

We use the classic solution derived for a homogeneous bracket band plate:

$$
\omega=\frac{\alpha}{2 \pi L^{2}} \sqrt{\frac{D_{e f f}}{\mu_{e f f}}},
$$

where $\alpha$ is defined by

$$
\cos (\alpha) e^{2 \alpha}+\cos (\alpha)+2 e^{\alpha}=0 .
$$


In this case, the hybrid method is the most accurate for the first three natural frequencies of the composite (Fig. 6). The accuracy is less than $1 \%$ for these frequencies. It should be noted that this type of asymmetric multiscale structure is very difficult to model by approximate methods.

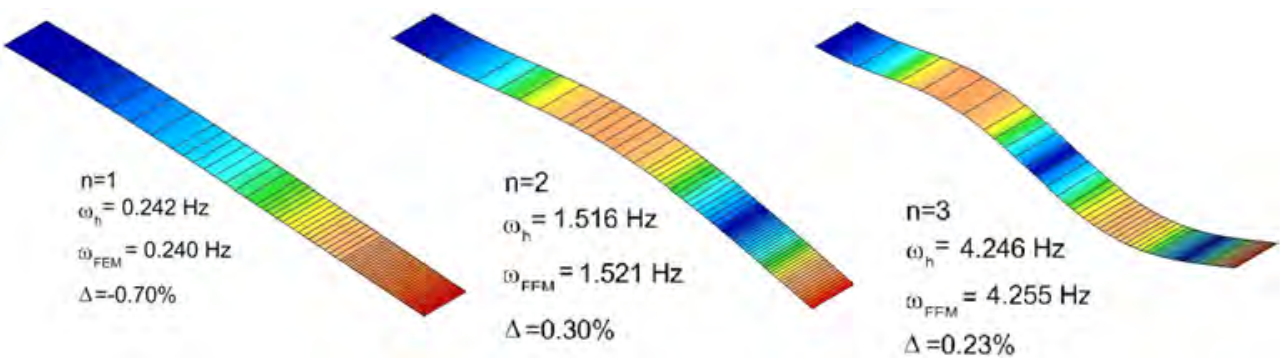

Fig. 6. Selected forms of free vibration for Example 2

For the entire frequency range, we can see that for the first ten vibration frequencies, the accuracy is always better than 5\%. As in Example 1, results from homogenization methods are underestimated.

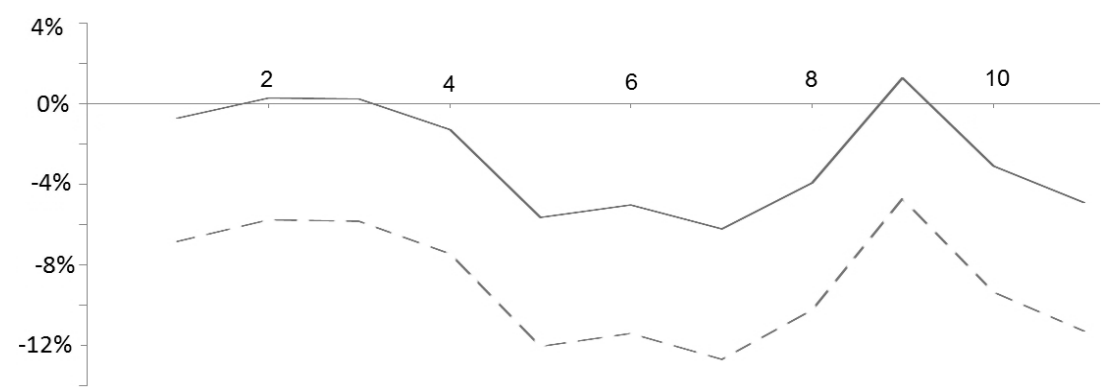

Fig. 7. Accuracy of solutions obtained from the proposed method (continuous line) and the asymptotic method (dashed line) in relation to the FEM (Example 2)

\subsection{Periodic structure - Example 3}

A slightly different situation occurs in the case of periodic systems. Here, due to the absence of multiscale effect the influence of the microstructure, size becomes visible only for the higher, above 10 order, free frequencies. Let us consider a simply supported plate band composed of three materials: glass, steel and aluminum of the total length $L=15 \mathrm{~m}$ and the thickness $h=0.1 \mathrm{~m}$ as shown in Figure 8 .

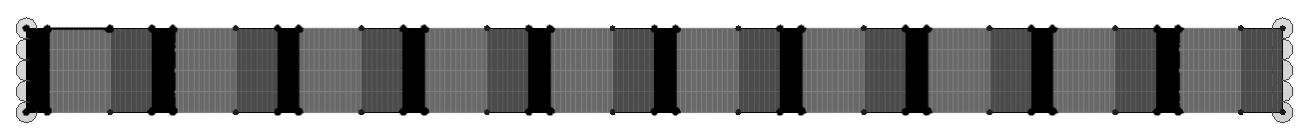

Fig. 8. Construction of the periodic band plate 
For such a proposed basic cell geometry, an antisymmetrical shape function has been taken in relation to the unit cell and the effective properties of the plate band have not been defined by formulas (24):

$$
\begin{gathered}
g=\sin \left(\frac{2 \pi x}{\lambda}\right),\left\langle D g_{, 11}\right\rangle=-54.8 \frac{\mathrm{MN}}{\mathrm{m}},\left\langle D g_{, 11} g_{, 11}\right\rangle=3330 \frac{\mathrm{MN}}{\mathrm{m}^{3}},\langle D\rangle=23 \mathrm{MNm}, \\
\langle\mu\rangle=608 \frac{\mathrm{kg}}{\mathrm{m}^{2}} .
\end{gathered}
$$

The plate band is simply supported:

$$
\left.\tilde{w}^{0}\right|_{x=a}=0,\left.\frac{\partial^{2} \tilde{w}^{0}}{\partial x^{2}}\right|_{x=a}=0,\left.\tilde{V}\right|_{x=a}=0,\left.\frac{\partial^{2} \tilde{V}}{\partial x^{2}}\right|_{x=a}=0, a=\{0, L\} .
$$

We use the classic solution derived for a homogeneous plate band

$$
\omega=\frac{k^{2} \pi}{2 L^{2}} \sqrt{\frac{D_{\text {eff }}}{\mu_{\text {eff }}}} .
$$

In this case we are not dealing with the multiscale structure, and the proposed hybrid method gives slightly worse results. Let us look at Figure 9. For the first eight free frequencies, the homogenization method gives much better results than the hybrid method. At around the ninth free frequency the accuracy of the homogenization method breaks down due to the increasing influence of the microstructure size. The hybrid method is more accurate for the $10^{\text {th }}-14^{\text {th }}$ frequencies.

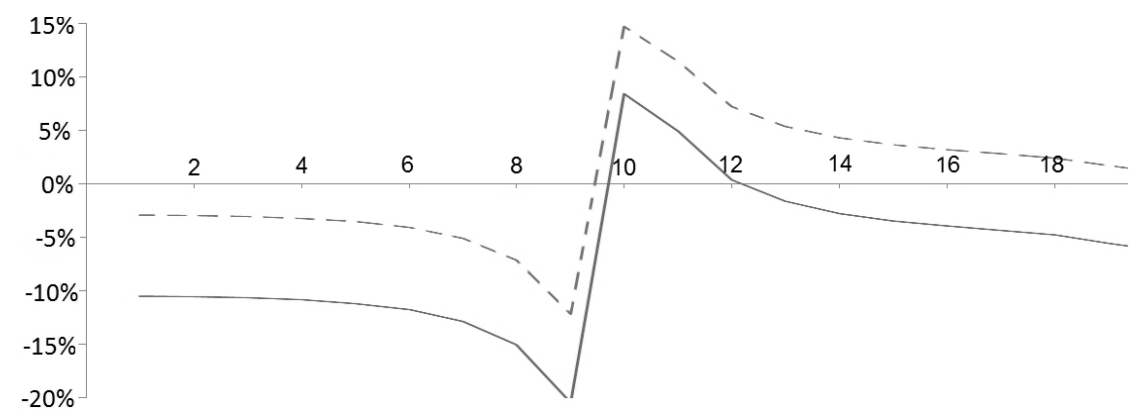

Fig. 9. Accuracy of solutions obtained from the proposed method (continuous line) and the asymptotic method (dashed line) in relation to the FEM

(periodic plate band) 


\section{Conclusions}

As a result of the presented modelling and numerical analysis, we can draw the following conclusions:

1. The presented hybrid method is best-suited for multiscale structures where the influence of the microstructure size is significant. In this case, the spectrum of the free vibration frequency of such a structure can successfully be obtained with a significantly better approximation than in the classic theory. The accuracy of this method in the presented examples of quasi-fractal structures was within the range of $5-7 \%$.

2. The obtained solutions are very simple and they can be used directly in engineering practice to estimate the values of the subsequent free vibration frequencies of quasi-fractal structures.

3. In case of periodic structures whose periodicity cell size is relatively small, it is recommended to use the homogenization methods as they are simpler and more accurate in relation to the first free vibration frequencies. Only in the case of the analysis of higher frequency vibrations, whose characteristic dimension of the form of vibrations is comparable to the microstructure size, can the presented hybrid model give more accurate results.

\section{References}

[1] Szymkiewicz A., Calculating effective conductivity of heterogeneous soils by homogenization, Archives of Hydro-Engineering and Environmental Mechanics 2005, 52, 2, 111-130.

[2] Woźniak C., Michalak B., Jędrysiak J. (eds.), Thermomechanics of Microheterogeneous Solids and Structures, Wydawnictwo Politechniki Łódzkiej, Łódź 2008.

[3] Vincent J.F.V., Bogatyreva O.A., Bogatyrev N.R., Bowyer A., Pahl A.-K., Biomimetics Its practice and theory, Journal of the Royal Society Interface 2006, 3(9), 471-482. doi:10.1098/rsif.2006.0127.

[4] Lévy-Véhel J., Lutton E. (eds.), Fractals in Engineering - New Trends in Theory and Applications, Springer, 2005.

[5] Lyons A., Materials for Architects and Builders, 4th Edition, Routledge, 2010.

[6] Woźniak C., Wierzbicki E., Averaging Techniques in Thermomechanics of Composite Solids, Wydawnictwo Politechniki Częstochowskiej, Częstochowa 2000.

[7] Michalak B., Analysis of dynamic behavior of wavy-plates with a mezzo-periodic structure, Journal of Theoretical and Applied Mechanics 2001, 39, 4, 947-958.

[8] Wirowski A., Self-vibration of thin plate band with non-linear functionally graded material, Archives of Mechanics 2012, 64, 6, 603-615.

[9] Ostrowski P., Michalak B., Non-stationary heat transfer in a hollow cylinder with functionally graded material properties, Journal of Theoretical and Applied Mechanics 2011, 49, 2, 385-397.

[10] Jędrysiak J., The length-scale effect in the buckling of thin periodic plates resting on a periodic Winkler foundation, Meccanica 2003, 38(4), 435-451. 